\title{
A METHOD FOR THE STATISTICAL EVALUATION OF CROSSTALK EFFECT BETWEEN THREE PARALLEL CONDUCTORS
}

\author{
P. T. TRAKADAS and C. N. CAPSALIS* \\ National Technical University of Athens, Department of Electrical \\ Engineering-Electroscience Division, Iroon Polytechniou 9, \\ str. Athens, Greece
}

(Received 11 January 1999; In final form 9 March 1999)

\begin{abstract}
There are several cases at which, in order to evaluate the crosstalk effect among transmission lines carrying useful signals, there is a need for probabilistic approach. This paper considers the problem of crosstalk estimation between transmission lines consisting of three conductors in a homogeneous surrounding medium, where the distance between the conductors is a random variable described by uniform distribution. The transmission lines are considered as electrically short. A closed-form equation is developed for the statistical distribution of the per-unit-length mutual inductance $\left(l_{m}\right)$ and an analytical one is described for the evaluation of the per-unit-length capacitance $\left(c_{m}\right)$. Theoretical results are compared with simulated ones for validation purposes.
\end{abstract}

Keywords: Crosstalk; statistical model; MTL method

\section{INTRODUCTION}

Nowadays, system designers are facing a paramount problem. In one hand, there are the market's needs for more power, greater bandwidth and very-large-scale integrated circuits. On the other hand, companies have to lower the manufacturing cost, increase the performance of their devices and deal with complex systems consisting of different kind of devices. In other words, distance of separation between transmission

*Corresponding author. Tel.: (+301) 772 2319, Fax: (+301) 7723557. 
lines - especially at PCBs - must be as small as possible in order to rake space on the substrate dimensions. On the contrary, the need for more power - resulting in higher-speed circuits - causes electromagnetic coupling between the cables. That is to say too many parameters must be considered during the manufacturing stage and be selected in such a way that all the requirements are satisfied. This is exactly where undesired mutual coupling effects (crosstalk) must be taken into account.

The general crosstalk problem involves multiple lines (ribbon cables) and complex geometries (PCBs) and is very complicated to analyze. In this paper, the crosstalk between three lossless, bare conductors in homogeneous medium is analyzed. Although this geometry is simple enough to analyze deterministically, the probabilistic approach is quite complicated. The significance of this paper is to give the setting for further analysis in more complicated geometries.

The crosstalk effect has been studied by many investigators [1,2]. A well-established deterministic model is explicitly described in [2], known as MTL (Multiconductor Transmission Lines) model. According to this model, matrix analysis is used to obtain deterministic models for the computation of $l_{m}$ and $c_{m}$. In [3], it is made clear that for electrically short lines, conductor losses can be neglected. In the last few years, many investigators using the previous model came to very useful results. In [4], C. R. Paul has shown how prediction of crosstalk in ribbon cables is affected by parameters such as the conductor losses, the dielectric insulation and the parasitic circuits, exhibiting experimental results compared to the prediction of the MTL model. The prediction accuracy for such frequencies for which the line is considered as electrically short $(L<\lambda / 10)$ is within $1 \mathrm{~dB}$, neglecting conductor losses. In [5], experimental data from more than 600 multi-pair cables are presented, showing that the gamma distribution is a more satisfactory approximation to model the multi-pair crosstalk behavior. In [6], a model for weakcoupled, uniform and non-uniform lines is presented. Measurements in a wide frequency range $(50 \mathrm{MHz}$ to $5 \mathrm{GHz}$ ) have shown good agreement with the theory described. In [7], a probabilistic model is developed in order to predict crosstalk effect between microstrip transmission lines. The model seems to have good agreement between the computed and the experimental measured. In [8], a probabilistic model has developed, based on the MTL model predicting the crosstalk effect on a specific geometry consisting of two parallel transmission lines above a ground 
plane whose distances are treated probabilistically. Good agreement between computed and measured results achieved.

The organization of this paper is as follows. Section II comprises of a description of the geometry under study and the fundamental expressions used in this paper. It also handles with the development of the statistical model of the per-unit-length mutual inductance and capacitance. In Section III, the developed models are validated by comparing the analytical results with simulated ones.

\section{THEORETICAL ANALYSIS OF THE PROBLEM}

\section{A. Deterministic Analysis of the Problem}

As it was mentioned before, a probabilistic approach for the evaluation of crosstalk effect between three parallel conductors will be derived. All three wires are considered to have radius equal to $r$ and perfect conductivity. The wire $O$ is the reference wire and is considered to be at a fixed position (Fig. 1). The other two wires are the driven wire (generator wire G) and the pickup wire (receptor wire R). Each of them forms a circuit with the reference wire. These two wires are considered to be moving uniformly along the $x$-axis. The distance between generator and reference conductor is equal to $d_{G}$ and the distance between the receptor and the reference is equal to $d_{R}$. The length of wires are thought to be too small compared to the dominant wavelength and the surrounding medium to be homogeneous $\left(\varepsilon_{0}, \mu_{0}\right)$. Consequently, the hypothesis of a pure TEM field structure is valid [2].

Using the theoretical analysis of the MTL model, in the frequency domain, for the certain geometry, one can easily derive [2] the

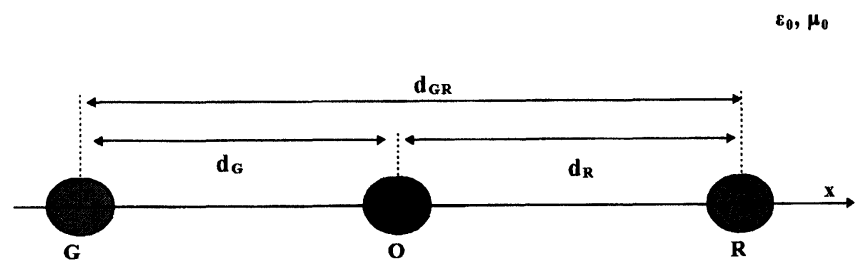

FIGURE 1 Geometry of the problem (P. T. Trakadas). 
expressions for the per-unit-length mutual inductance and capacitance and the self inductance of the receptor and generator wire, written below:

$$
\begin{gathered}
l_{G}=\frac{\mu_{0}}{2 \pi} \cdot \ln \left(\frac{d_{G}^{2}}{r^{2}}\right) \\
l_{R}=\frac{\mu_{0}}{2 \pi} \cdot \ln \left(\frac{d_{R}^{2}}{r^{2}}\right) \\
l_{m}=\frac{\mu_{0}}{2 \pi} \cdot \ln \left(\frac{d_{G} \cdot d_{R}}{r \cdot d_{G R}}\right) \\
c_{m}=\frac{1}{u^{2}} \cdot \frac{l_{m}}{l_{G} \cdot l_{R}-l_{m}^{2}}
\end{gathered}
$$

where: $r$ is the radius of wires,

$\mu_{0}$ is the magnetic permeability for free space, $u$ is the velocity of waves on the line.

\section{B. Statistical Distribution of $l_{m}$}

Suppose the independent variables $d_{G}, d_{R}$ as random, with uniform distributions. It is of great importance to set the minimum distance between the wires larger than $4 r$. This condition provides that charge and current distributions are uniform. The maximum distance is $b$ $(b>4 r)$. The pdf of each one is considered in the form [9]:

$$
\begin{gathered}
f_{d_{G}}\left(d_{G}\right)=\frac{1}{b-4 \cdot r}, \quad 4 \cdot r \leq d_{G} \leq b \\
f_{d_{R}}\left(d_{R}\right)=\frac{1}{b-4 \cdot r}, \quad 4 \cdot r \leq d_{R} \leq b
\end{gathered}
$$

These two random variables are statistically independent. The joint pdf can be expressed as:

$$
f_{d_{G}, d_{R}}\left(d_{G}, d_{R}\right)=f_{d_{G}}\left(d_{G}\right) \cdot f_{d_{R}}\left(d_{R}\right)
$$


As it is obvious from (1c), $l_{m}$ depends on the values of $d_{G}, d_{R}$ and $d_{G R}$ $\left(d_{G R}=d_{G}+d_{R}\right)$. In order to determine the density of $l_{m}$, first the following transformation is used:

$$
\begin{gathered}
z_{1}=\frac{\mu_{0}}{2 \cdot \pi} \cdot \ln \left(\frac{d_{G} \cdot d_{R}}{r \cdot\left(d_{G}+d_{R}\right)}\right) \\
z_{2}=d_{G}
\end{gathered}
$$

Then,

$$
f_{z_{1}, z_{2}}\left(z_{1}, z_{2}\right)=\left.\frac{f_{d_{G}}\left(d_{G}\right) \cdot f_{d_{R}}\left(d_{R}\right)}{\left|J\left(d_{G}, d_{R}\right)\right|}\right|_{\substack{d_{G}=z_{2} \\ d_{R}=d_{R}\left(z_{1}, z_{2}\right)}}
$$

where:

$$
\left|J\left(d_{G}, d_{R}\right)\right|=\left|\begin{array}{ll}
\frac{\partial z_{1}}{\partial d_{G}} & \frac{\partial z_{1}}{\partial d_{R}} \\
\frac{\partial z_{2}}{\partial d_{G}} & \frac{\partial z_{2}}{\partial d_{R}}
\end{array}\right|=\frac{\mu_{0} \cdot d_{G}}{2 \cdot \pi \cdot d_{R} \cdot\left(d_{G}+d_{R}\right)}
$$

Then, the density of $z_{1}$ (or $\left.l_{m}\right)$ is given as:

$$
f_{z_{1}}\left(z_{1}\right)=\int_{-\infty}^{\infty} f_{z_{1}, z_{2}}\left(z_{1}, z_{2}\right) \cdot d z_{2}
$$

Using (2a), (2b), (4d) and (4c), follows:

$$
f_{z_{1}, z_{2}}\left(z_{1}, z_{2}\right)=\frac{2 \cdot \pi \cdot d_{R} \cdot\left(d_{R}+z_{2}\right)}{\mu_{0} \cdot(b-4 \cdot r)^{2} \cdot z_{2}}
$$

where:

$$
d_{R}=\frac{r \cdot z_{2} \cdot \exp \left(2 \cdot \pi \cdot z_{1} / \mu_{0}\right)}{z_{2}-r \cdot \exp \left(2 \cdot \pi \cdot z_{1} / \mu_{0}\right)}
$$

The random variables $d_{R}, d_{G}\left(=z_{2}\right)$ are limited to $[4 r, b]$ as well as $z_{1}$ is limited to:

$$
\left[\frac{\mu_{0} \cdot \ln 2}{2 \cdot \pi}, \frac{\mu_{0} \cdot \ln \left(\frac{b}{2 \cdot r}\right)}{2 \cdot \pi}\right]
$$


The minimum value of $z_{1}$ comes up for $\left(d_{G}, d_{R}\right)=(4 r, 4 r)$ and the maximum for $\left(d_{G}, d_{R}\right)=(b, b)$.

In order to evaluate pdf of $z_{1}$ (Eq. (5)), one must find the equation giving $z_{2}$ as a function of $z_{1}$ and $d_{R}$. From Eq. (6b):

$$
z_{2}=\frac{r \cdot d_{R} \cdot \exp \left(\frac{2 \cdot \pi \cdot z_{1}}{\mu_{0}}\right)}{d_{R}-r \cdot \exp \left(\frac{2 \cdot \pi \cdot z_{1}}{\mu_{0}}\right)}
$$

Figure 2 shows the curves of $z_{2}$ for the minimum and maximum values of $d_{R}$ and for the domain of $z_{1}$.

where:

$$
\zeta_{1}=\frac{\mu_{0} \cdot \ln 2}{2 \cdot \pi}, \quad \zeta_{2}=\frac{\mu_{0} \cdot \ln \left(\frac{4 \cdot b}{b+4 \cdot r}\right)}{2 \cdot \pi}, \quad \zeta_{3}=\frac{\mu_{0} \cdot \ln \left(\frac{b}{2 \cdot r}\right)}{2 \cdot \pi}
$$

It is obvious that $z_{2}$ is a branchy function:

$$
z_{2}=\left\{\begin{array}{l}
\frac{4 \cdot r \cdot a\left(z_{1}\right)}{4-a\left(z_{1}\right)}, \frac{\mu_{0}}{2 \cdot \pi} \cdot \ln (2) \leq z_{1} \leq \frac{\mu_{0}}{2 \cdot \pi} \cdot \ln \left(\frac{4 \cdot b}{b+4 \cdot r}\right), d_{R}=4 \cdot r \\
\frac{r \cdot b \cdot a\left(z_{1}\right)}{b-r \cdot a\left(z_{1}\right)}, \frac{\mu_{0}}{2 \cdot \pi} \cdot \ln \left(\frac{4 \cdot b}{b+4 \cdot r}\right) \leq z_{1} \leq \frac{\mu_{0}}{2 \cdot \pi} \cdot \ln \left(\frac{b}{2 \cdot r}\right), d_{R}=b
\end{array}\right.
$$

where $\alpha\left(z_{1}\right)=\exp \left(2 \pi z_{1} / \mu_{0}\right)$.
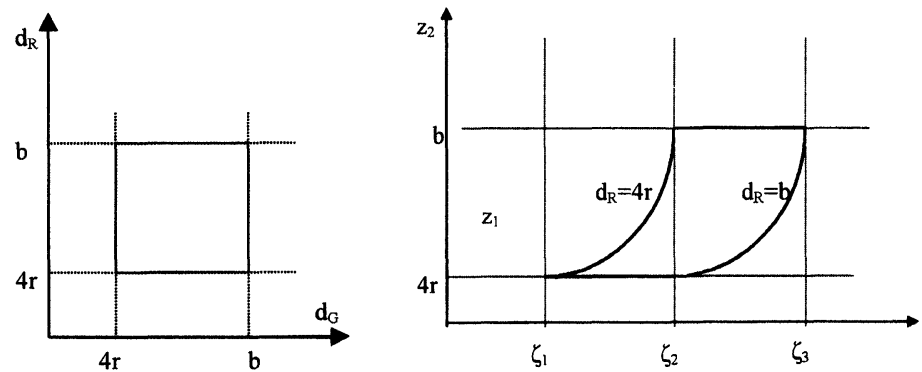

FIGURE 2 Transformation of the $\left(d_{G}, d_{R}\right)$ domain to $\left(z_{1}, z_{2}\right)$ domain (P. T. Trakadas). 
Knowing the expression giving $z_{2}$ as a function of $z_{1}$, one can evaluate the density of $l_{m}$ (Eq. (5e)):

$f_{l_{m}}\left(l_{m}\right)=f_{z_{1}}\left(z_{1}\right)=\left\{\begin{array}{l}\int_{4 r}^{h_{1}\left(z_{1}\right)} f_{z_{1}, z_{2}}\left(z_{1}, z_{2}\right) \cdot d z_{2}, \frac{\mu_{0}}{2 \cdot \pi} \cdot \ln 2 \leq z_{1} \leq \frac{\mu_{0}}{2 \cdot \pi} \cdot \ln \left(\frac{4 \cdot b}{b+4 \cdot r}\right), \\ d_{R}=4 \cdot r \\ \int_{h_{2}\left(z_{1}\right)}^{b} f_{z_{1}, z_{2}}\left(z_{1}, z_{2}\right) \cdot d z_{2}, \frac{\mu_{0}}{2 \cdot \pi} \cdot \ln \left(\frac{4 \cdot b}{b+4 \cdot r}\right) \leq z_{1} \leq \frac{\mu_{0}}{2 \cdot \pi} \cdot \ln \left(\frac{b}{2 \cdot r}\right), \\ d_{R}=b\end{array}\right.$

where

$$
h_{1}\left(z_{1}\right)=\frac{4 \cdot r \cdot \exp \left(\frac{2 \cdot \pi \cdot z_{1}}{\mu_{0}}\right)}{4-\exp \left(\frac{2 \cdot \pi \cdot z_{1}}{\mu_{0}}\right)}, \quad h_{2}\left(z_{1}\right)=\frac{r \cdot b \cdot \exp \left(\frac{2 \cdot \pi \cdot z_{1}}{\mu_{0}}\right)}{b-r \cdot \exp \left(\frac{2 \cdot \pi \cdot z_{1}}{\mu_{0}}\right)}
$$

The joint pdf $f_{z_{1}}, f_{z_{2}}\left(z_{1}, z_{2}\right)$ is given in (6a).

Carrying out the calculations, the probability density function of $l_{m}$ is given analytically as:

$$
f_{l_{m}}\left(l_{m}\right)=\left\{\begin{array}{l}
\frac{4 \cdot \pi \cdot r^{2} \cdot a\left(l_{m}\right)}{\mu_{0} \cdot(b-4 \cdot r)^{2} \cdot\left(4-a\left(l_{m}\right)\right)} \\
\quad\left[-16+8 \cdot a\left(l_{m}\right)+\left(4 \cdot a\left(l_{m}\right)-a^{2}\left(l_{m}\right)\right)\right. \\
\left.\quad \cdot\left(2 \cdot \ln a\left(l_{m}\right)-2 \cdot \ln \left(4-a\left(l_{m}\right)\right)\right)\right] \\
\frac{\mu_{0}}{2 \cdot \pi} \cdot \ln 2 \leq l_{m} \leq \frac{\mu}{2 \cdot \pi} \cdot \ln \left(\frac{4 \cdot b}{b+4 \cdot r}\right) \\
\frac{4 \cdot \pi \cdot r \cdot a\left(l_{m}\right)}{\mu_{0} \cdot(b-4 \cdot r)^{2} \cdot\left(b-r \cdot a\left(l_{m}\right)\right)} \\
{\left[b^{2}-2 \cdot b \cdot r \cdot a\left(l_{m}\right)+\left(r^{2} \cdot a^{2}\left(l_{m}\right)-b \cdot r \cdot a\left(l_{m}\right)\right)\right.} \\
\left.\quad \cdot\left(2 \cdot \ln a\left(l_{m}\right)+2 \cdot \ln \left(\frac{r}{b-r \cdot a\left(l_{m}\right)}\right)\right)\right] \\
\frac{\mu_{0}}{2 \cdot \pi} \cdot \ln \left(\frac{4 \cdot b}{b-4 \cdot r}\right) \leq l_{m} \leq \frac{\mu_{0}}{2 \cdot \pi} \cdot \ln \left(\frac{b}{2 \cdot r}\right)
\end{array}\right.
$$

where

$$
a\left(l_{m}\right)=\exp \left(\frac{2 \cdot \pi \cdot l_{m}}{\mu_{0}}\right)
$$

\section{Statistical Distribution of $c_{m}$}

The equation giving the per-unit-length mutual capacitance is written at (1d). Substituting Eqs. (1a), (1b), (1c) into (1d), one can easily express 
$c_{m}$ as a function of independent variables $d_{G}, d_{R}$, given below:

$$
c_{m}=\frac{1}{u^{2}} \cdot \frac{\frac{\mu_{0}}{2 \pi} \cdot \ln \left(\frac{d_{G} \cdot d_{R}}{r \cdot\left(d_{G}+d_{R}\right)}\right)}{\left(\frac{\mu_{0}}{\pi}\right)^{2} \ln \frac{d_{G}}{r} \cdot \ln \frac{d_{R}}{r}-\left(\frac{\mu_{0}}{2 \pi}\right)^{2} \cdot \ln ^{2}\left(\frac{d_{G} \cdot d_{R}}{r \cdot\left(d_{G}+d_{R}\right)}\right)}
$$

Because of the fact that this equation is transcendental, a closed form solution of the probability density function of $c_{m}$ is extremely difficult to find.

Finally, using an auxiliary variable $w=d_{G}$, the pdf of $c_{m}$ can be written:

$$
f_{c_{m}}\left(c_{m}\right)=\left.\int_{4 r}^{b} \frac{f_{d_{G}}\left(d_{G}\right) \cdot f_{d_{R}}\left(d_{R}\right)}{\left|\frac{\partial c_{m}}{\partial d_{R}}\right|} \cdot d w\right|_{\substack{w=d_{G} \\ d_{R}=d_{R}\left(c_{m}, w\right)}}
$$

\section{COMPARISON BETWEEN COMPUTED AND SIMULATED RESULTS}

In this section simulated results are presented and compared with the theoretical results for $l_{m}$ and $c_{m}$. For the completeness of the paper, it is mentioned that the radius of the wires was taken equal to $50 \mu \mathrm{m}$, maximum distance between the wires $b=1 \mathrm{~mm}$ and the velocity $u=3 \cdot 10^{8}$ $\mathrm{m} / \mathrm{sec}$. In Figure 3, the cumulative density function for both analytical and simulated [10] results are given. An excellent agreement is observed.

In Figure 4, the simulated cdf of $c_{m}$ is presented.

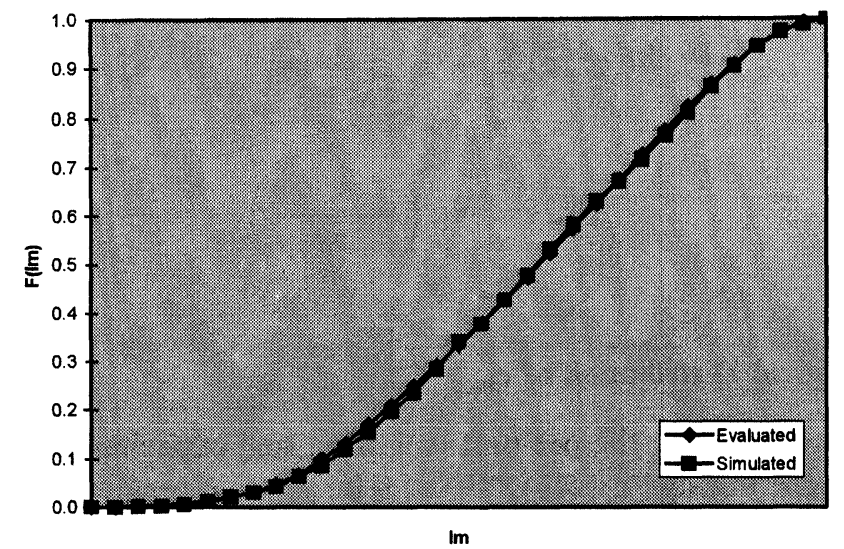

FIGURE 3 Comparison between simulated and evaluated cdf of $l_{m}$. 


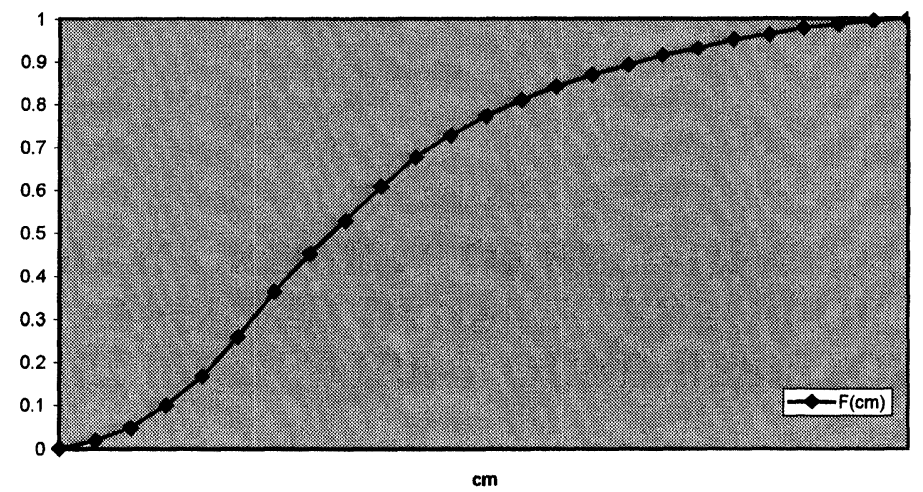

FIGURE 4 Simulated cdf of $c_{m}$.

\section{CONCLUSIONS}

In this paper, a probabilistic model has been presented for the estimation of crosstalk between three parallel conductors. Closed-form and analytical results derived for the evaluation of the cumulative density function of the per-unit-length mutual inductance and capacitance, respectively, and compared with simulated results. The method can be used in order to analyze more complicated structures.

\section{References}

[1] Weston, D. A. (1991). "Electromagnetic Compatibility, Principles and Applications", Marcel Dekker, Inc.

[2] Paul, C. R. (1991). "Introduction to Electromagnetic Compatibility", John Wiley and Sons, Inc.

[3] Cory, H. and Shiran, S., "The Importance of Losses in the Coupling of Transmission Lines", IEEE Trans. EMC, 30(4), Nov. 1988, 567-570.

[4] Paul, C. R. (1978). "Prediction of Crosstalk in Ribbon Cables: Comparison of Model Predictions and Experimental Results", IEEE Trans. EMC, EMC-20(3), Aug. 1978, 394-406.

[5] Lin, S. H., "Statistical Behaviour of Multipair Crosstalk", Bell System Technical Journal, 59(6), July-August 1980, pp. 955-974.

[6] Hill, D., Canvey, K. and Johnk, R., "Crosstalk Between Microstrip Transmission Lines", IEEE Trans. EMC, 36(4), Nov. 1994, 314-321.

[7] Linares, R. M., Kontorovich, V. and Jardon, H., "A Probabilistic Model for the Prediction of Crosstalk in PCBs", Int. Symp. on Electromagn. Comp., Rome, Italy, Sept. 17-20, pp. 621-626.

[8] Shiran, S., Reiser, B. and Cory, H., "A Probabilistic Model for the Evaluation of Coupling Between Transmission Lines", IEEE Trans. EMC, 35(3), Aug. 1993, 387-393.

[9] Papoulis, A. (1981). "Probability, Random Variables and Stochastic Processes", New York, McGraw Hill.

[10] Wolfram, S. (1996). "The Mathematica Book, version 3", Cambridge University Press. 

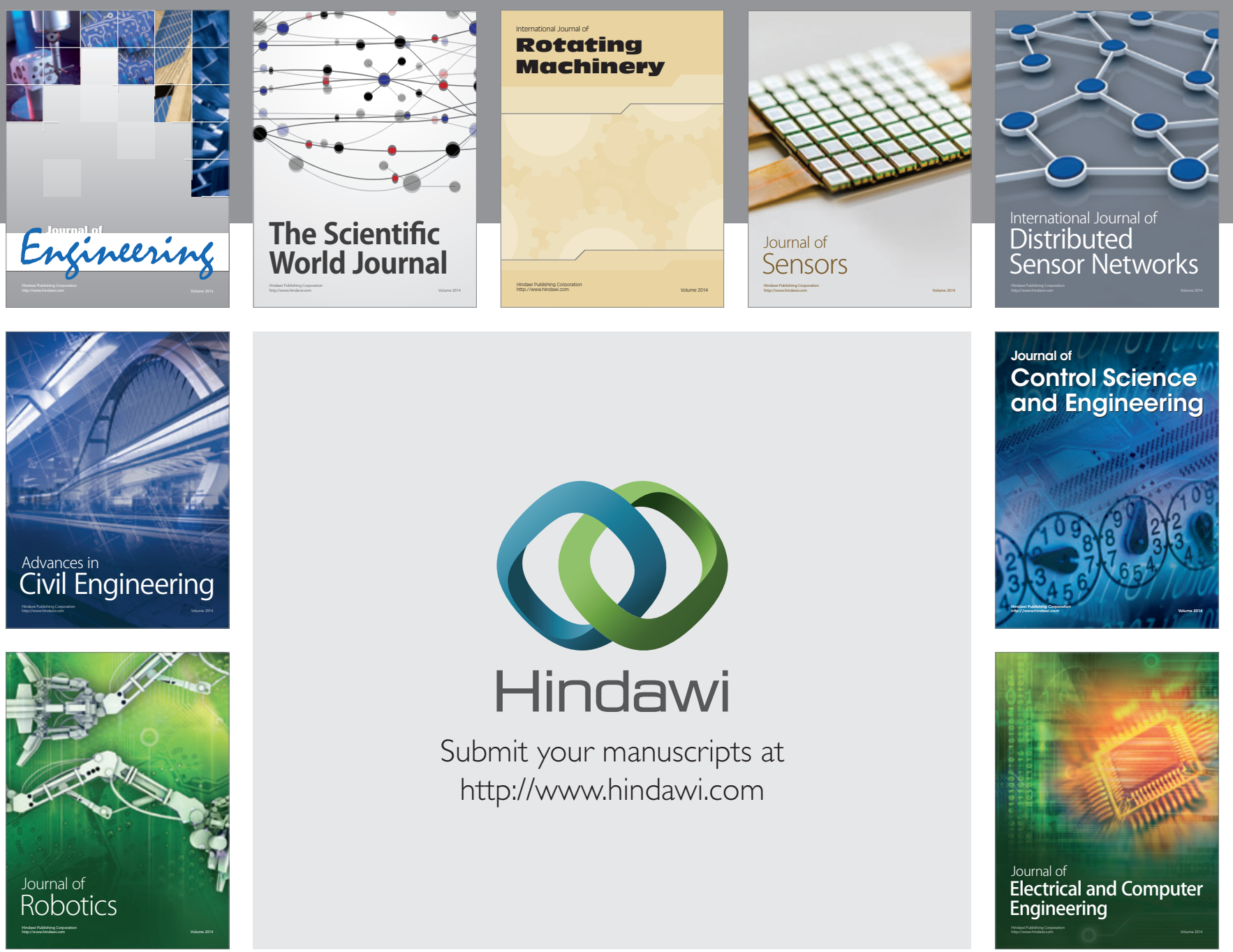

Submit your manuscripts at

http://www.hindawi.com
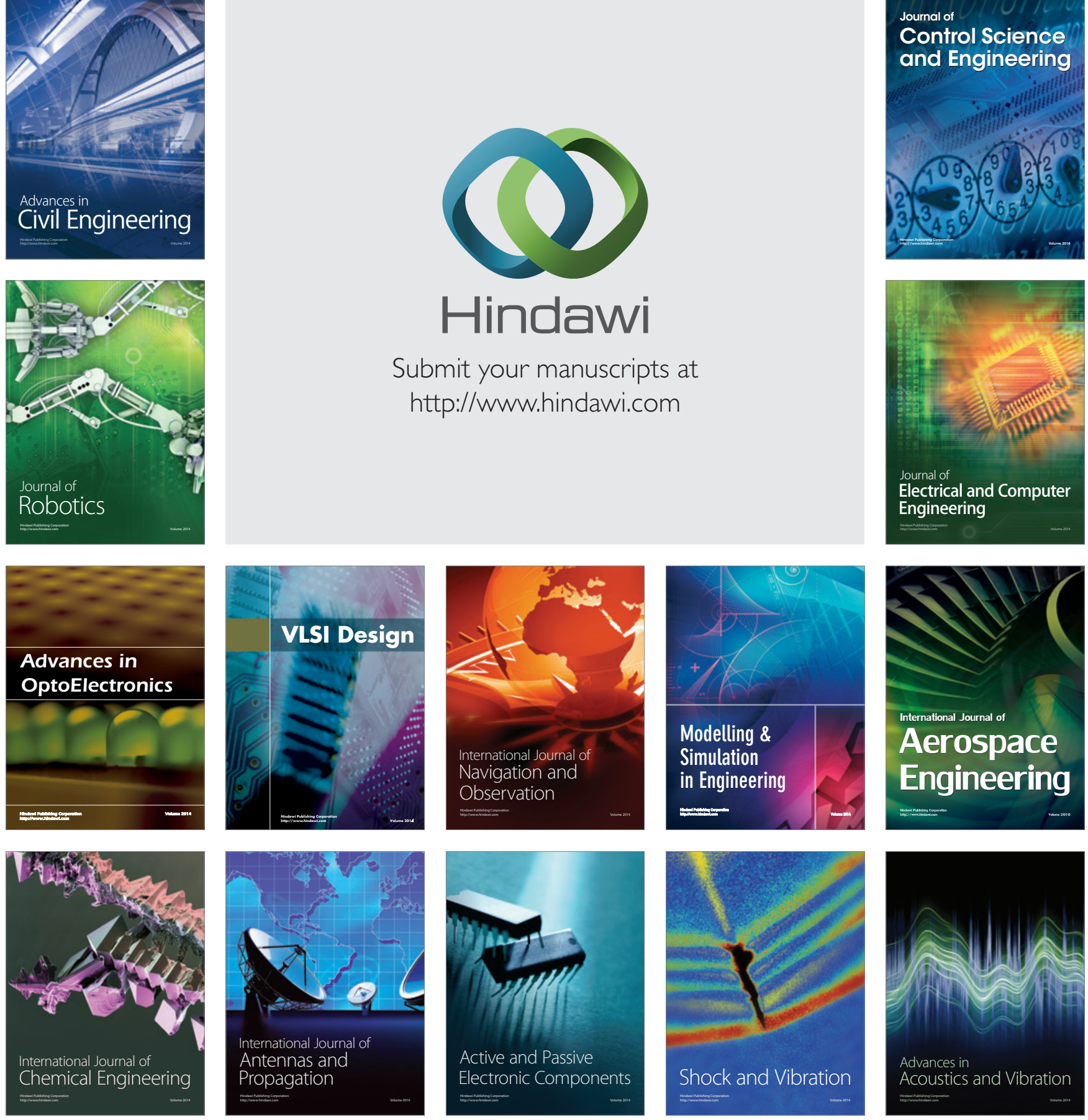